\title{
Évaluation de l'exposition de la population française à la radioactivité naturelle
}

\author{
S. BILLON ${ }^{1}$, A. MORIN ${ }^{1}$, S. CAËR ${ }^{2}$, H. BAYSSON ${ }^{1}$, J.P. GAMBARD ${ }^{1}$, \\ A. RANNOU ${ }^{1}$, M. TIRMARCHE ${ }^{\prime}$, D. LAURIER ${ }^{\prime}$
}

(Manuscrit reçu le 24 septembre 2003, accepté le 18 janvier 2004)

RÉSUMÉ L'exposition aux rayonnements ionisants de la population française est due en majeure partie à la radioactivité naturelle (radon, irradiations externes d'origine tellurique et cosmique, ingestion d'eau et d'aliments). Dans un contexte épidémiologique, il est nécessaire d'estimer au mieux l'exposition de la population afin de pouvoir l'étudier conjointement à des indicateurs sanitaires. Dans cet objectif, des indicateurs d'exposition doivent être créés en prenant en compte les mesures environnementales et en contrôlant les facteurs de variabilité (caractéristiques de l'habitat, variations saisonnières, densité de population). La distribution des expositions doit également pouvoir être étudiée à différents niveaux géographiques (département, zone d'emploi). Le présent travail constitue une mise à jour de l'estimation de l'exposition de la population française à la radioactivité naturelle. Ies indicateurs d'exposition au radon domestique reposent sur les concentrations mesurées, corrigées pour la saison et les caractéristiques d'habitat (variation départementale: $19-297 \mathrm{~Bq} / \mathrm{m}^{3}$ ). Les indicateurs d'exposition au rayonnement gamma d'origine tellurique reposent sur les débits de dose mesurés, redressés sur le type d'habitat (22-95 $\mathrm{nSv} / \mathrm{h})$. L'exposition aux rayonnements cosmiques est évaluée à partir de l'altitude et pondérée sur la densité de population $(0,27-0,38 \mathrm{mSv} / \mathrm{an})$. La dose efficace annuelle due à ces trois composantes est estimée à $2,2 \mathrm{mSv}$.

ABSTRACT Evaluation of natural radiation exposure of the French population.

Exposure of the French population to ionising radiation is main!y due to natural radiation (i.e. exposure through: inhalation of radon decay products, external radiation of terrestrial and cosmic origin and water and food ingestion). In an epidemiological context, it is necessary to estimate as precisely as possible the population exposure, in order to study its influence on health indicators. In this aim, indicators of population exposure should be created taking into account results of environmental measurements by controlling the different factors that may influence these measurements (dwelling characteristics, seasonal variations, population density). The distribution of these exposures should also be studied at different geographical levels (department, job area). This work updates the estimation of the French population exposure to natural radiation. Radon exposure indicators have been based on concentrations measured in dwellings, corrected on season and dwelling characteristics (departmental range: $19-297 \mathrm{~Bq} / \mathrm{m}^{3}$ ). Indicators of terrestrial gamma ray exposure have been based on measured indoor and outdoor dose rates adjusted on dwelling characteristics (22-95 nSv/h). Cosmic ray exposure

\footnotetext{
I IRSN, DRPH, Lahoratoire d'épidémiologie. B.P. 17, 92262 Fontenay-aux-Roses Cedex. France.

2 Centre d'assurance de qualité des applications technologiques dans le domaine de la sunté, 92340 Bourg-la-Reine. France.
} 
has been evaluated from altitude and weighted by population density (0.27$0.38 \mathrm{mSv} / \mathrm{yr}$ ). Due to these three components, the effective annual dose was estimated to be at $2.2 \mathrm{mSv}$.

\section{Introduction}

Les deux principales sources d'exposition de la population française aux rayonnements ionisants sont les irradiations pour raisons médicales $(41 \%)$ et les irradiations d'origine naturelle $(58 \%)$, auxquelles viennent s'ajouter les irradiations d'origine industrielle ( $1 \%$ ). L'exposition à la radioactivité naturelle est due pour $59 \%$ au radon, $19 \%$ aux rayonnements gamma d'origine tellurique (rayonnement des sols), $12 \%$ aux rayonnements cosmiques et $10 \%$ à l'eau et aux aliments (Sugier et Hubert, 2002). Cependant cette répartition masque une variabilité importante de l'exposition individuelle à la radioactivité naturelle en fonction de la localisation géographique, des caractéristiques de l'habitat, de la saison, etc.

En épidémiologie, une estimation moyenne nationale de l'exposition de la population est rarement suffisante lorsqu'il s'agit de mettre en parallèle des données d'exposition avec des données sanitaires, notamment à cause de la grande variabilité de certains indicateurs par régions (ou départements). Il est donc nécessaire d'obtenir une estimation la plus précise possible. Pour répondre à cet objectif, les mesures brutes ont été corrigées pour les principaux facteurs pouvant influencer cette exposition. Une estimation de la distribution géographique de l'exposition à la radioactivité naturelle peut être ainsi décrite, en tenant compte de la variabilité intra-régions (ou intra-départements), notamment pour réaliser des études écologiques. D’une manière générale, celles-ci ont pour but d'étudier la relation entre les caractéristiques collectives d'exposition relevées sur plusieurs populations et les valeurs de risque de maladie observées au sein de ces mêmes populations. Les unités statistiques des études écologiques ne sont pas des individus mais des groupes de populations.

Les principaux facteurs susceptibles de modifier les concentrations en radon dans l'habitat sont : la géologie sous-jacente, la saison, le matériau de construction de l'habitat, le type d'habitat (pavillon/immeuble), la localisation de la pièce mesurée (rez-de-chaussée/étage) et à un moindre degré, la période de construction, la ventilation (ou isolation) et le mode de chauffage (Arvela, 1995 ; Bochicchio et al., 2002 ; Gunby et al., 1993 ; Kim et al., 2003 ; Kitto, 2003 ; Majborn, 1992 ; Miles, 2001; Pirard et al., 1998 ; Verger et al., 1994). Les conditions atmosphériques (vitesse du vent, pression atmosphérique, humidité...) participent également à la modification des concentrations en radon (Buchli et Burkart, 1985 ; Miles, 2001). 
Les principaux facteurs de variabilité (intra- et inter-département) des débits de dose des rayonnements gamma d'origine tellurique sont : la géologie sous-jacente, le matériau de construction, le type d'habitat et la période de construction (Idrish Miah, 2001 ; Iyogi et àl., 2002 ; Mjönes, 1986 ; Rannou et al., 1984).

Les principaux facteurs de variabilité des doses dues aux rayonnements cosmiques sont l'altitude et l'activité solaire.

La prise en compte de la distribution de la densité de population permet de calculer une exposition moyenne pondérée par les populations exposées à un endroit donné à la radioactivité naturelle. Les départements à densité de population élevée contribuent ainsi davantage à l'estimation de l'exposition moyenne nationale que les départements avec une densité de population faible.

Les effets de la radioactivité naturelle sur la santé ont fait l'objet de nombreuses études, en particulier leur lien avec certains cancers (Axelson et al., 2002 ; Baysson et Tirmarche, 2004 ; Baysson et al., 2004 ; Darby et al., 2001 ; Flodin et al., 1990 ; Forastiere et al., 1998 ; Kendall et Smith, 2002 ; Laurier et al., 2001 ; Rommens et al., 2001 ; Tirmarche et al., 1988). L'objectif de la présente étude est de fournir des estimateurs de l'exposition de la population française aux différentes sources de radioactivité naturelle (radon, irradiations externes d'origine tellurique et cosmique), afin de les mettre en parallèle, dans de futures études, avec des indicateurs de surveillance sanitaire, par exemple avec l'incidence des leucémies chez l'enfant ou la mortalité par cancer du poumon chez l'adulte. Le présent article apporte des éléments complémentaires et est donc une réactualisation et une synthèse des études françaises antérieures (Billon et al., 2003 ; Chartier et al., 1997 ; Gambard et al., 2000 ; Rannou et al., 1984 ; Verger et al., 1994).

\section{Matériel et méthodes}

\subsection{Données d'exposition de la population à la radioactivité naturelle}

La campagne nationale de mesure de la concentration du radon domestique en France a été conduite par l'Institut de radioprotection et de sûreté nucléaire (IRSN) en collaboration avec la Direction générale de la santé (DGS) et les Directions départementales des affaires sanitaires et sociales (DDASS), avec pour objectif l'étude de la distribution du radon dans l'habitat privé français (Gambard et al., 2000). Les principaux objectifs étaient d'identifier les zones à haut potentiel radon, de définir le pourcentage d'habitats au-dessus des normes et de mettre en évidence les facteurs modifiant les concentrations en radon (Gambard et al., 2000 ; Pirard et al., 1998). Les mesures dans l'habitat ont été effectuées avec un détecteur Kodalpha LR 115 installé pendant deux mois dans la pièce principale. De 1982 
à 1991, la recherche des habitations où poser les dosimètres avait été laissée à 1'appréciation des organismes concernés. Depuis 1992, la sélection des habitations dans un département s'appuyait sur un double critère démographique et géographique (méthode du maillage), le but étant d'obtenir une couverture homogène de la surface du département (Gambard et al., 2000). Concernant la population, environ un point de mesure a été effectué pour 1500 habitants; pratiquement, une mesure a été effectuée dans chaque commune de plus de 500 habitants en zone rurale ou entre 1000 et 1500 habitants en zone urbaine. Au plan géographique, la couverture ne devait pas présenter une trop grande hétérogénéité (c'est-à-dire trop de grandes zones sans mesure); 100 à 200 points de mesure étaient en général suffisants pour couvrir un département. À chaque mesure était associé un questionnaire visant à mettre en évidence les facteurs influant sur les concentrations en radon (type d'habitat, période de construction...). Les données utilisées dans cette étude sont celles des précédentes publications (Gambard et al., 2000 ; Pirard et al., 1998 ; Verger et al., 1994) complétées avec des mesures plus récentes pour certains départements (jusqu'en mars 2002). Parmi les 13324 mesures sur l'ensemble du territoire métropolitain, seules celles collectées en dehors des caves et des sous-sols, avec une durée de mesure connue, supérieure à un mois et inférieure ou égale à un an ont été conservées pour ce travail, soit 12261 mesures et une couverture nationale de 10098 communes.

Une campagne de mesure des débits de dose des irradiations externes d'origine tellurique et cosmique a également été menée en France par l'IRSN, la DGS et les DDASS, de 1977 à 1990 et de 1996 à 2002, dans le but d'estimer la distribution des doses externes (Rannou et al., 1992). Les mesures ont été faites à l'intérieur de l'habitat et à l'extérieur au moyen de différents appareils de mesure suivant la période considérée: tube de sulfate de calcium, dosimètre thermoluminescent PGP1 et dosimètre PANASONIC (durées de pose de 6 mois, de 1977 à 1990) et radiamètre SAPHYMO (durée de mesure de 45 minutes, de 1996 à 2002, uniquement à l'intérieur de l'habitat). À partir de 1996, les mesures de débit de dose des rayonnements gamma ont été faites à l'intérieur de l'habitat en parallèle aux mesures de la concentration du radon. À chaque mesure était associé un questionnaire identique à celui de la campagne de mesure du radon. 14031 mesures de débit de dose dû aux irradiations externes ont été effectuées, dont 8737 mesures à l'intérieur de l'habitat, concernant 5739 communes dans 59 départements et 5294 mesures à l'extérieur de l'habitat, concernant 3269 communes dans 38 départements.

Par ailleurs, les doses efficaces moyennes annuelles dues aux rayonnements cosmiques ont été calculées en s'appuyant sur les travaux du Comité scientifique des Nations-Unies pour l'étude des effets des rayonnements ionisants 
(UNSCEAR, 2000) et en utilisant les données d'altitude de l'Institut géographique national (IGN), pour l'ensemble des communes françaises (Morin et al., 2002).

Les débits de dose mesurés dus aux irradiations externes englobent la contribution des rayonnements d'origine tellurique et d'origine cosmique. Pour obtenir les débits de dose dus aux rayonnements gamma d'origine tellurique, les débits de dose dus aux rayonnements cosmiques ont été calculés à partir de l'altitude de la commune puis déduits des débits de dose mesurés dus aux irradiations externes. Le facteur d'atténuation des matériaux de 0,8 a également été pris en compte pour les mesures à l'intérieur de l'habitat, comme le préconise l'UNSCEAR (2000).

\subsection{Estimation de l'exposition de la population à la radioactivité naturelle}

Le traitement de l'ensemble de ces données a permis leur description détaillée (caractéristiques statistiques, représentations cartographiques).

Afin de fournir des indicateurs d'exposition de la population les plus réalistes possible, les données de concentrations et de débits de dose ont été croisées avec des données démographiques (densité de population, distribution par type de logement) et des indicateurs de comportement, provenant essentiellement de l'Institut national de la statistique et des études économiques (INSEE).

Concernant les concentrations en radon mesurées à l'intérieur des habitations, il existe une variation selon la saison de mesure, se traduisant par des concentrations maximales en hiver et minimales en été (Pinel et al., 1995). Du fait que les concentrations en radon ont été mesurées pendant une durée de deux mois, une correction est nécessaire afin d'estimer le niveau de concentration annuelle et donc de rendre les concentrations comparables entre elles. La méthodologie est détaillée par Baysson et al. (2003).

Les habitats mesurés à l'occasion des campagnes ne sont pas représentatifs du parc immobilier français: en effet, les mesures ont été privilégiées dans les pavillons. Les données «logement - emploi - population » du recensement général de l'INSEE de 1990 fournissent le nombre de logements suivant leur type (pavillon ou immeuble) et leur période de construction (avant ou après 1948), par commune. Ces données, également recueillies dans les questionnaires des campagnes de mesures, ont été utilisées comme facteur de pondération afin d'estimer une exposition représentative du parc immobilier français. Pour chaque département, les concentrations en radon et les débits de dose des rayonnements gamma d'origine tellurique ont été stratifiés en fonction des quatre catégories 
TABLEAU I

Moyennes des caractéristiques statistiques départementales des expositions au radon et aux irradiations externes d'origine tellurique et cosmique.

Averages of departmental statistical characteristics of exposure to radon and external radiation of terrestrial or cosmic origin.

\begin{tabular}{|c|c|c|c|c|}
\hline \multicolumn{5}{|l|}{ 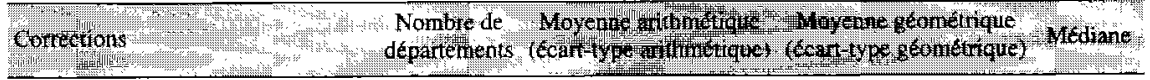 } \\
\hline \multicolumn{5}{|l|}{ Radon $\left(\mathrm{Bq} / \mathrm{m}^{3}\right)$} \\
\hline - allocune & 96 & $89(115)$ & $59(2)$ & 55 \\
\hline - saison & 96 & $87(113)$ & $58(2)$ & 54 \\
\hline - salson et logement & 94 & $83(-)$ & - & - \\
\hline $\begin{array}{l}\text { - saison, logement er densité } \\
\text { de population }\end{array}$ & 94 & $63(-)$ & - & - \\
\hline \multicolumn{5}{|c|}{ Rayonnements gamma d'origine tellurique ( $\mathrm{nSv} / \mathrm{h}$ ) } \\
\hline - intérieur : aucune & 59 & $55(17)$ & $52(1)$ & $53 \ldots$ \\
\hline - inténeter : logement & 59 & $54(-)$ & - & ; \\
\hline $\begin{array}{l}\text { - intérieur : logement et densité } \\
\text { de population }\end{array}$ & $\cdots 59$ & $53(+)$ & - & (n) \\
\hline * extérieur : aucune & 38 & $46(14)$ & $44(1)$ & 45 \\
\hline - extérieur : densitê de population & 38 & $46(-)$ & $=$ & $\therefore$ \\
\hline \multicolumn{5}{|l|}{ Rayonnements cosmiques (mSv/an) } \\
\hline - aucune & 96 & $0,29(0,07)$ & $0,29 \cdot(1,04)$ & 0,28 \\
\hline - densité de population & 96 & $0,28(0,01)$ & $0,28(1,03)$ & 0.28 \\
\hline
\end{tabular}

d'habitat ainsi obtenues (pavillon ancien, pavillon récent, immeuble ancien et immeuble récent) et une moyenne a été calculée pour chacune de ces strates. L'exposition moyenne dans chaque département est donc la somme pondérée de ces quatre moyennes sur la proportion de la catégorie d'habitat correspondante recensée dans le département concerné (Billon et al., 2002).

Les données du recensement général de la population de l'INSEE de 1999 ont été utilisées pour fournir une moyenne nationale pondérée sur la densité de population de chaque département, pour le radon et les rayonnements gamma d'origine tellurique (Billon et al., 2002), et des moyennes départementales pondérées sur la densité de population de chaque commune, pour les rayonnements cosmiques (Morin et al., 2002).

Par ailleurs, dans un but de gestion du risque, l'exposition de la population française à la radioactivité naturelle peut s'exprimer en dose efficace annuelle. 
La dose efficace due à la radioactivité naturelle est alors la somme de différents indicateurs d'exposition de la population, pondérés sur la durée de présence à l'intérieur des bâtiments, estimée à environ $90 \%$ par l'étude CIBLEX (BeaugelinSeiler et al., 2002).

\section{Résultats}

\subsection{Exposition au radon domestique}

Les 12261 mesures de concentration en radon dans l'habitat français ont une moyenne arithmétique de 89 becquerels par mètre cube $\left(\mathrm{Bq} / \mathrm{m}^{3}\right.$ ) (écart-type : $162 \mathrm{~Bq} / \mathrm{m}^{3}$ ) et une moyenne géométrique de $53 \mathrm{~Bq} / \mathrm{m}^{3}$ (écart-type géométrique : $3 \mathrm{~Bq} / \mathrm{m}^{3}$ ). Au niveau départemental, les moyennes arithmétiques brutes varient de $22 \mathrm{~Bq} / \mathrm{m}^{3}$ à Paris à $263 \mathrm{~Bq} / \mathrm{m}^{3}$ en Corse-du-Sud (Tab. I, Fig. 1). La moyenne arithmétique corrigée pour la saison de mesure est de $87 \mathrm{~Bq} / \mathrm{m}^{3}$. La correction pour la saison de mesure et pour les caractéristiques de l'habitat a été faite pour 94 départements. La perte de deux départements est due à la présence de valeurs manquantes pour les caractéristiques de l'habitat dans l'Hérault et le Tarn. La moyenne arithmétique corrigée est alors de $83 \mathrm{~Bq} / \mathrm{m}^{3}$, les moyennes départementales variant de $19 \mathrm{~Bq} / \mathrm{m}^{3}$ à Paris à $297 \mathrm{~Bq} / \mathrm{m}^{3}$ en Lozère (Tab. I, Fig. 2). La moyenne arithmétique nationale corrigée pour la saison, le logement et la densité de population diminue à $63 \mathrm{~Bq} / \mathrm{m}^{3}$, du fait que les départements les plus peuplés présentent en général des concentrations moyennes basses.

\subsection{Exposition aux rayonnements gamma d'origine tellurique}

Les 14031 débits de dose dus aux rayonnements gamma d'origine tellurique ont une moyenne arithmétique brute de 55 nanosievert par heure $(\mathrm{nSv} / \mathrm{h})$ (écart-type : $18 \mathrm{nSv} / \mathrm{h}$ ) à l'intérieur de l'habitat pour 59 départements, et de $46 \mathrm{nSv} / \mathrm{h}$ (écart-type : $15 \mathrm{nSv} / \mathrm{h}$ ) à l'extérieur de l'habitat pour 38 départements. Au niveau départemental, les moyennes arithmétiques brutes varient, à l'intérieur de l'habitat, de $23 \mathrm{nSv} / \mathrm{h}$ dans le Jura à $96 \mathrm{nSv} / \mathrm{h}$ dans l'Allier et à l'extérieur de l'habitat, de $25 \mathrm{nSv} / \mathrm{h}$ dans le Vaucluse à $85 \mathrm{nSv} / \mathrm{h}$ dans l'Allier (Tab. I, Figs. 3 et 4).

Les variations associées aux caractéristiques de l'habitat sont bien moindres que pour les concentrations en radon: à l'intérieur de l'habitat, la moyenne arithmétique corrigée pour le logement et la densité de population est de $53 \mathrm{nSv} / \mathrm{h}$. À l'extérieur de l'habitat, la moyenne arithmétique pondérée sur la densité de population est de $46 \mathrm{nSv} / \mathrm{h}$, soit quasiment inchangée par rapport à la moyenne brute. 


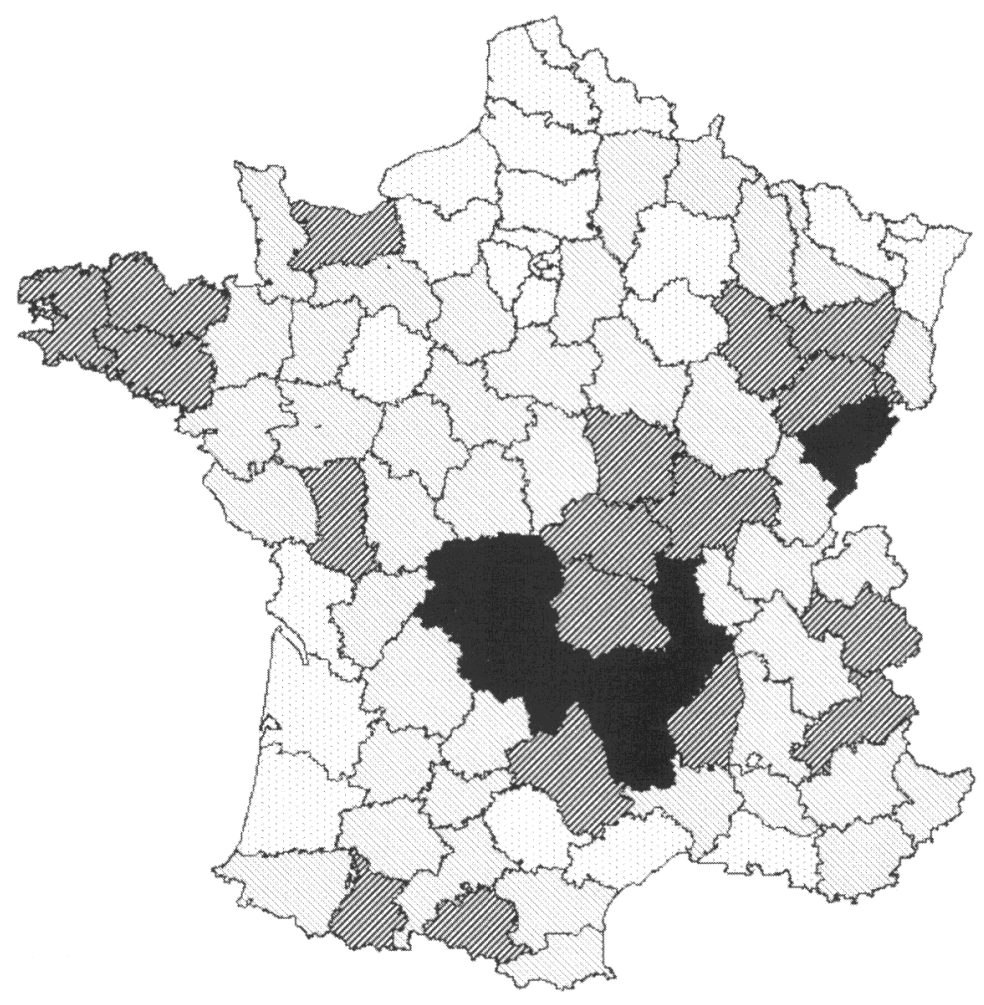

Concentrations en radon, en $\mathrm{Bq} / \mathrm{m}^{3}-$ Radon concentrations, in $\mathrm{Bq} / \mathrm{m}^{3}$ :

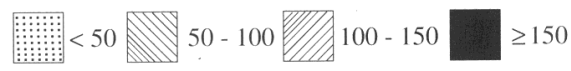

Figure 1 - Moyennes arithmétiques des concentrations en radon par département, en $\mathrm{Bq} / \mathrm{m}^{3}$. Arithmetic means of radon concentrations by department, in $\mathrm{Bq} / \mathrm{m}^{3}$. 


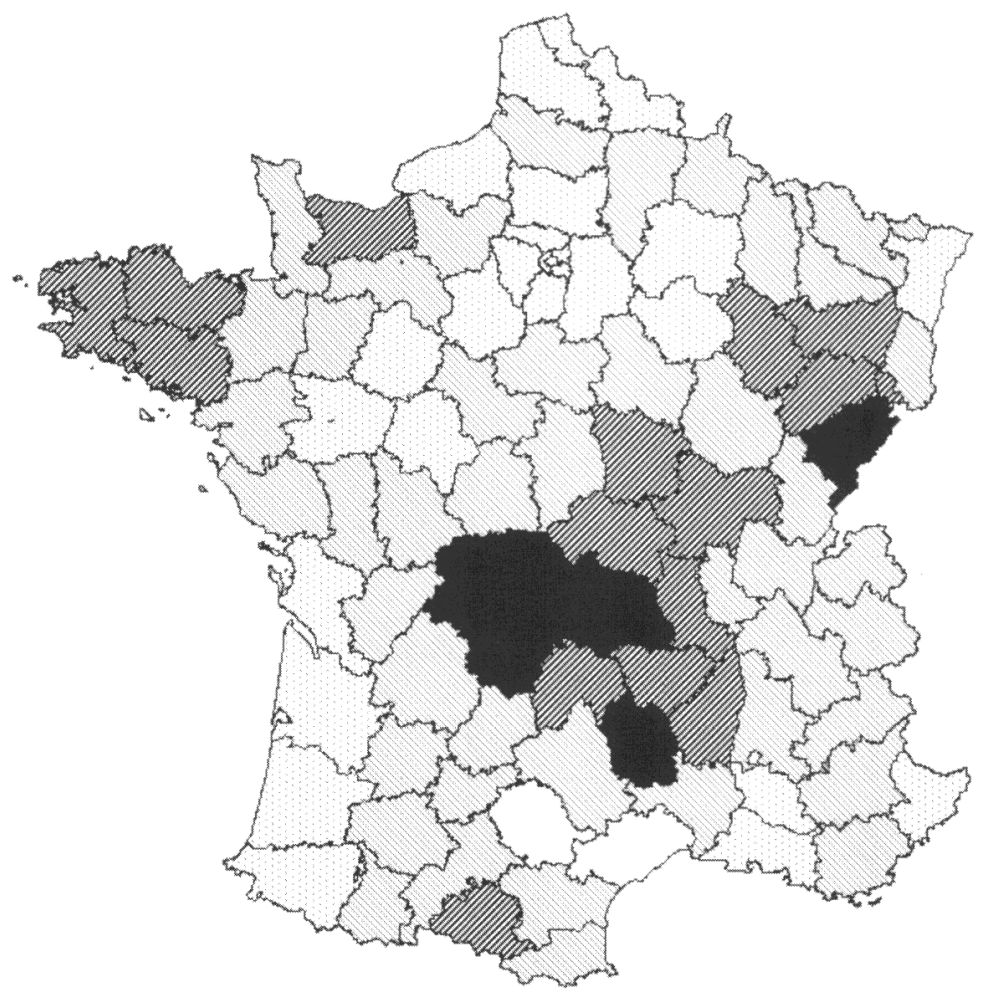

Concentrations en radon, en $\mathrm{Bq} / \mathrm{m}^{3}$ - Radon concentrations, in $\mathrm{Bq} / \mathrm{m}^{3}$ :

W $<50 \geqq 50-100 \square 100-150 \square \geq 150 \square$ Manquant - Missing

Figure 2-Moyennes arithmétiques des concentrations en radon par département, en $\mathrm{Bq} / \mathrm{m}^{3}$, après correction pour la saison et le type d'habitat.

Arithmetic means of radon concentrations by department, in $\mathrm{Bq} / \mathrm{m}^{3}$, after season and dwelling type corrections. 

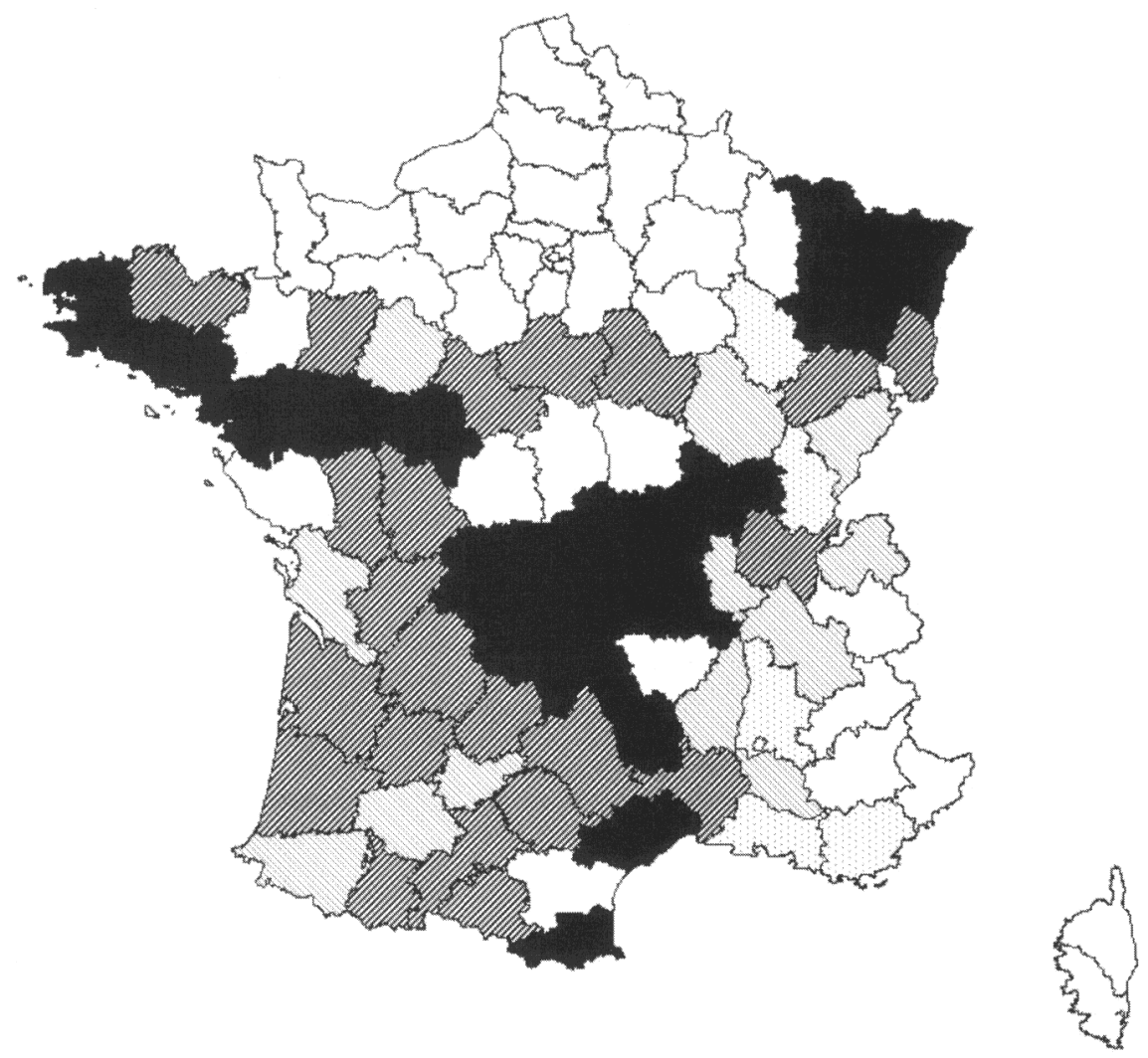

Débits de dose des rayonnements gamma, en nSv/h - Gamma rays dose rates, in $n S v / h$ :

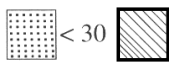

$30-45$

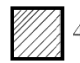

$45-60$
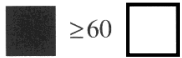

Manquant - Missing

Figure 3 - Moyennes arithmétiques des débits de dose des rayonnements gamma d'origine tellurique par département (en $n S v / h$ ), à l'intérieur de l'habitat.

Arithmetic means of indoor terrestrial gamma radiation dose rate in air by department (in $n S v / h$ ). 


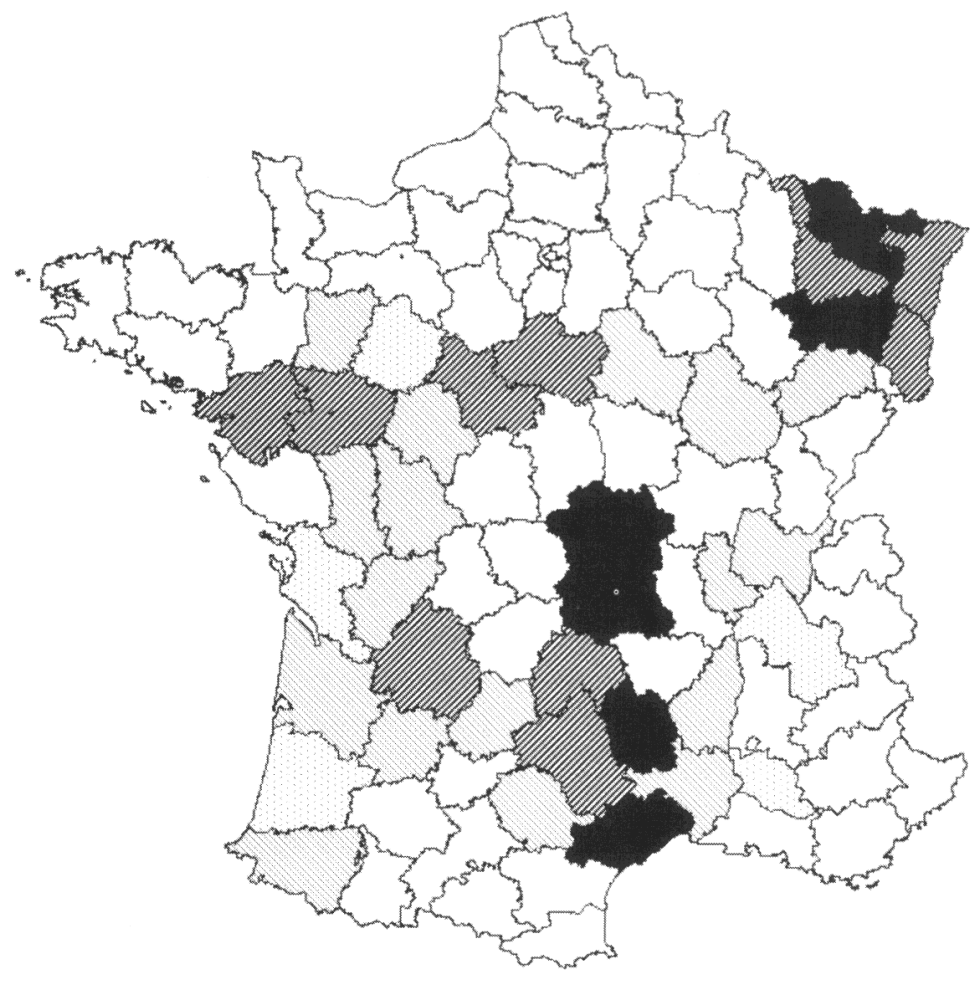

Débits de dose des rayonnements gamma, en $\mathrm{nSv} / \mathrm{h}$ - Gamma rays dose rates, in $n S v / h$ :

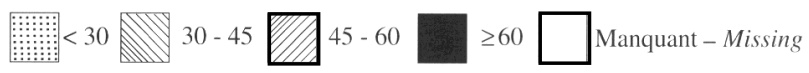

Figure 4-Moyennes arithmétiques des débits de dose des rayonnements gamma d'origine tellurique par département (en $n \mathrm{~Sv} / \mathrm{h}$ ), à l'extérieur de l'habitat.

Arithmetic means of outdoor terrestrial gamma radiation dose rate in air by department (in $n S v / h$ ). 


\subsection{Exposition aux rayonnements cosmiques}

Après pondération sur la densité de population des communes et prise en compte du temps passé à l'intérieur des bâtiments ( $90 \%$ du temps), la dose efficace annuelle moyenne due aux rayonnements cosmiques varie selon les départements entre 0,27 (dans 53 départements situés à une altitude proche du niveau de la mer) à 0,38 millisievert par an (mSv/an) dans les Hautes-Alpes (Tab. I, Fig. 5), avec une moyenne arithmétique de $0,28 \mathrm{mSv} / \mathrm{an}$.

\section{Discussion et perspectives}

\subsection{Limites et avantages}

Les campagnes nationales de mesure des concentrations en radon et des débits de dose dus aux irradiations externes ont commencé à la fin des années 1970. L'hypothèse est faite que les modes de vie des populations et les caractéristiques de l'habitat ont relativement peu changé au cours des trois dernières décennies et que, par conséquent, les concentrations et débits de dose mesurés reflètent l'exposition moyenne de la population française.

Au vu de la localisation des mesures, les concentrations mesurées fournissent une couverture plus ou moins représentative de l'exposition de la population. La campagne de mesure des concentrations en radon dans l'habitat se poursuit actuellement afin de compléter les départements couverts de manière hétérogène (notamment la Côte d'Or, l'Hérault, la Manche et le Tarn). Quant aux débits de dose des rayonnements gamma d'origine tellurique, les données disponibles ne permettent pas de couvrir la totalité du territoire français, mais seulement deux tiers du territoire (59 départements couverts sur 96, essentiellement au sud de la France). Une première solution serait de relancer la campagne de mesures dans les départements manquants. Une seconde solution serait d'utiliser les autres données gamma existant en France, collectées par l'ex-Office de protection contre les rayonnements ionisants (OPRI, récemment intégré à l'IRSN). Ces données sont issues de mesures de 6 mois effectuées à l'aide de dosimètres thermoluminescents (TLD) et qui portent sur l'ensemble du territoire ou encore de mesures instantanées effectuées par des balises Téléray dans des préfectures ou sous-préfectures, des centrales nucléaires, des aéroports, des sites OPRI ou des sommets (OPRI, 2001). Ces mesures sont effectuées dans un but d'alerte et de surveillance environnementale et non dans l'optique d'évaluer l'exposition des populations. Par conséquent, les débits de dose mesurés par les TLD et par les balises Téléray sont disponibles principalement à l'extérieur de l'habitat. Une étude complémentaire devrait donc être faite sur ces données de surveillance environnementale, afin d'établir la 


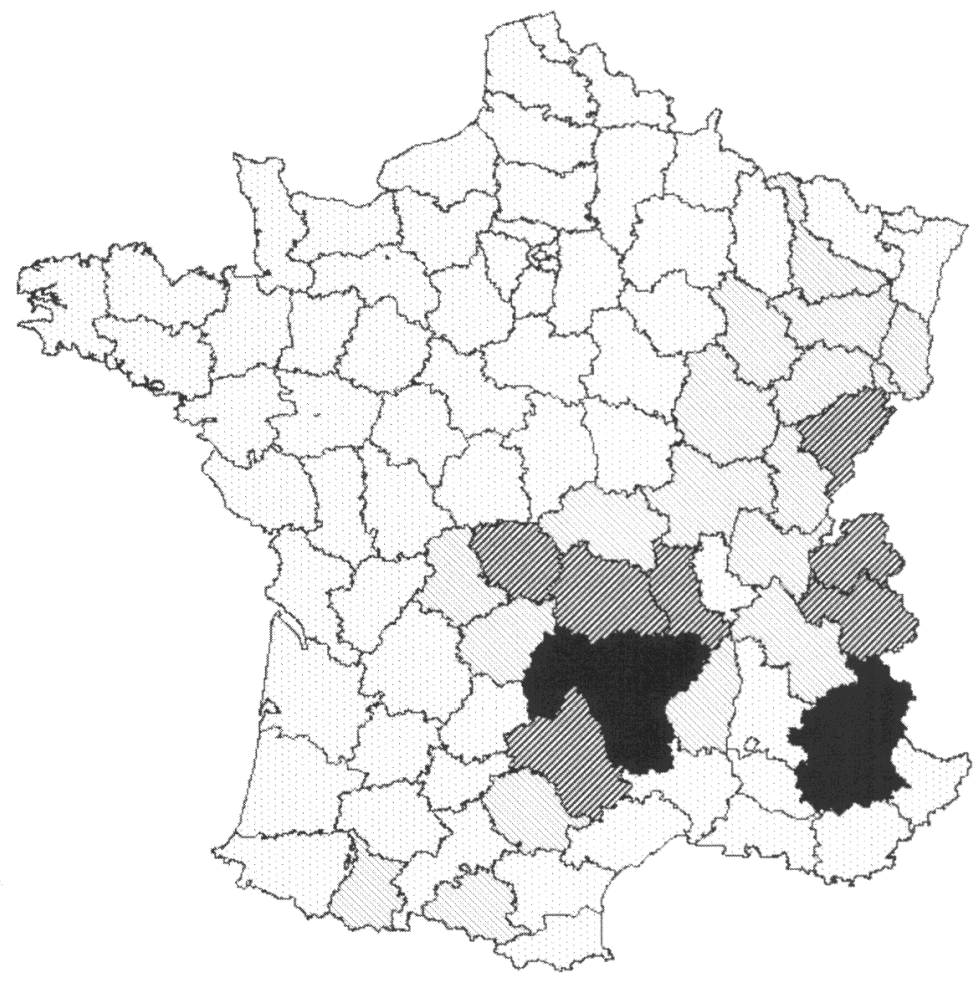

Dose efficace due aux rayonnements cosmiques, en $\mathrm{mSv} / \mathrm{an}$ - Effective dose due to cosmic rays, in $\mathrm{mSv} / \mathrm{yr}$ :

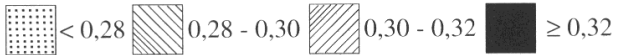

Figure 5 - Dose efficace moyenne due aux rayonnements cosmiques par département (en mSv/an), après correction sur la densité de population.

Effective dose due to cosmic radiation by department (in $\mathrm{mSv} / \mathrm{yr}$ ), after population density correction. 
meilleure façon de les intégrer aux données d'exposition aux rayonnements gamma.

La présente étude a l'avantage de reposer sur des bases de données de taille importante (plus de 12000 points de mesure, chacune). Ces données permettent d'avoir une bonne représentation de la distribution géographique de l'exposition à la radioactivité naturelle, ainsi que de la variabilité intra-département de l'exposition au radon et aux rayonnements cosmiques (sur toute la France), ainsi qu'aux rayonnements gamma d'origine tellurique (sur les 59 départements couverts). La France présente la caractéristique d'avoir des variations géographiques importantes des niveaux de radon et des irradiations externes d'origine tellurique et cosmique, de par sa géologie complexe et ses reliefs. Les cartes (Figs. 1-5) permettent d'illustrer la distribution géographique de la radioactivité naturelle et d'identifier les zones à forte variation.

L'estimation de l'exposition de la population à la radioactivité naturelle a été faite sur des données d'exposition corrigées pour les facteurs d'influence suivants : la saison pour le radon, les caractéristiques de l'habitat (pavillon ou immeuble, et construit avant ou après 1948) pour le radon et les rayonnements gamma d'origine tellurique, et également la densité de population pour les trois sources d'exposition étudiées. D'autres facteurs d'influence mis en évidence dans la littérature n'ont pas pu être pris en compte dans cette estimation de l'exposition des populations au radon : c'est le cas par exemple de la géologie, du niveau d'étage, du matériau de construction, de l'isolation (ou ventilation) et du mode de chauffage de l'habitat mesuré. Plusieurs facteurs peuvent jouer sur les variabilités inter-habitat, mais n'ont pas pu être pris en compte à cause de données manquantes associées aux mesures ou à cause de données qui n'existent pas pour la population française. Des facteurs comme la géologie et le niveau d'étage sont partiellement pris en compte dans le découpage géographique et les corrections pour le type d'habitat, respectivement. Il est toutefois nécessaire d'aller plus loin pour effectuer des évaluations locales ou pour estimer des données manquantes. L'activité solaire n'a pas été prise en compte de manière directe dans l'estimation de l'exposition aux rayonnements cosmiques. Cependant, la dose annuelle estimée peut être considérée comme un indicateur moyen de l'exposition aux rayonnements cosmiques sur plusieurs années, qui tient compte indirectement des fluctuations de l'activité solaire d'une année sur l'autre.

La présente étude apporte plusieurs améliorations par rapport aux études antérieures menées en France (Billon et al., 2003 ; Chartier et al., 1997 ; Gambard et al., 2000 ; Rannou et al., 1984 ; Verger et al., 1994). L'une de ces améliorations est l'augmentation de la taille des bases de données : les données d'exposition au radon sont plus complètes, notamment pour 5 départements (les Alpes-Maritimes, 
la Drôme, la Haute-Garonne, la Haute-Saône et les Vosges) ; de plus, elles ont été sélectionnées de manière à conserver uniquement les mesures relatives à des lieux de vie pour l'estimation de l'exposition de la population. Une autre amélioration est que la prise en compte de l'analyse des facteurs d'influence (type d'habitat, période de construction, saison) et l'utilisation de données extérieures, en particulier provenant de l'INSEE, permettent de fournir des estimateurs a priori plus proches de l'exposition réelle de la population.

Une finalité de cette étude peut être l'estimation de l'exposition de la population au niveau d'un découpage administratif plus fin que les départements ou les régions, et utilisé pour des indicateurs sanitaires, tels que les zones d'emploi (Fig. 6, exposition au radon). Une zone d'emploi est un espace géographique à l'intérieur duquel la plupart des actifs résident et travaillent. Suite à la révision de 1994, la France est découpée en 348 zones homogènes vis-à-vis de critères sociodémographiques. D’autres finalités sont la cartographie des niveaux moyens d'exposition par département, et également l'estimation de la variabilité inter et intra unité géographique (département, zone d'emploi), ce qui est très important pour la mise en parallèle avec des indicateurs sanitaires.

En terme de résultats, l'impact des corrections appliquées est plus important pour l'exposition au radon que pour l'exposition aux irradiations externes. L'impact des corrections est relativement faible au niveau de la représentation cartographique des expositions départementales, mais il est particulièrement important lors de l'estimation de l'exposition nationale tenant compte de la localisation géographique de la population.

\subsection{Dose efficace}

Dans une optique de gestion du risque, il peut être utile de connaître la dose efficace due à la radioactivité naturelle. Néanmoins, à ce stade de notre étude, la part due à l'ingestion (notamment via l'eau et les aliments) n'a pas été réalisée.

L'étude CIBLEX (Beaugelin-Seiler et al., 2002) a montré que la population française passe en moyenne $92,6 \%$ de son temps à l'intérieur de bâtiments (de 91,9\% en Provence-Alpes-Côte-d'Azur et en Corse à 93,5\% en île-de-France). Une étude anglaise (BBC, 1978) concluait à un résultat très proche, avec un temps passé à l'intérieur des bâtiments de $90 \%$, par opposition au niveau mondial de $80 \%$ (UNSCEAR, 2000). Le calcul de dose efficace a donc été fait en prenant un facteur d'occupation de $90 \%$ à l'intérieur des bâtiments. En supposant que l'exposition reçue à l'intérieur des bâtiments professionnels est du même ordre de grandeur que l'exposition reçue à l'intérieur de l'habitat privé, la dose efficace annuelle moyenne due à la radioactivité naturelle peut être estimée par la somme 

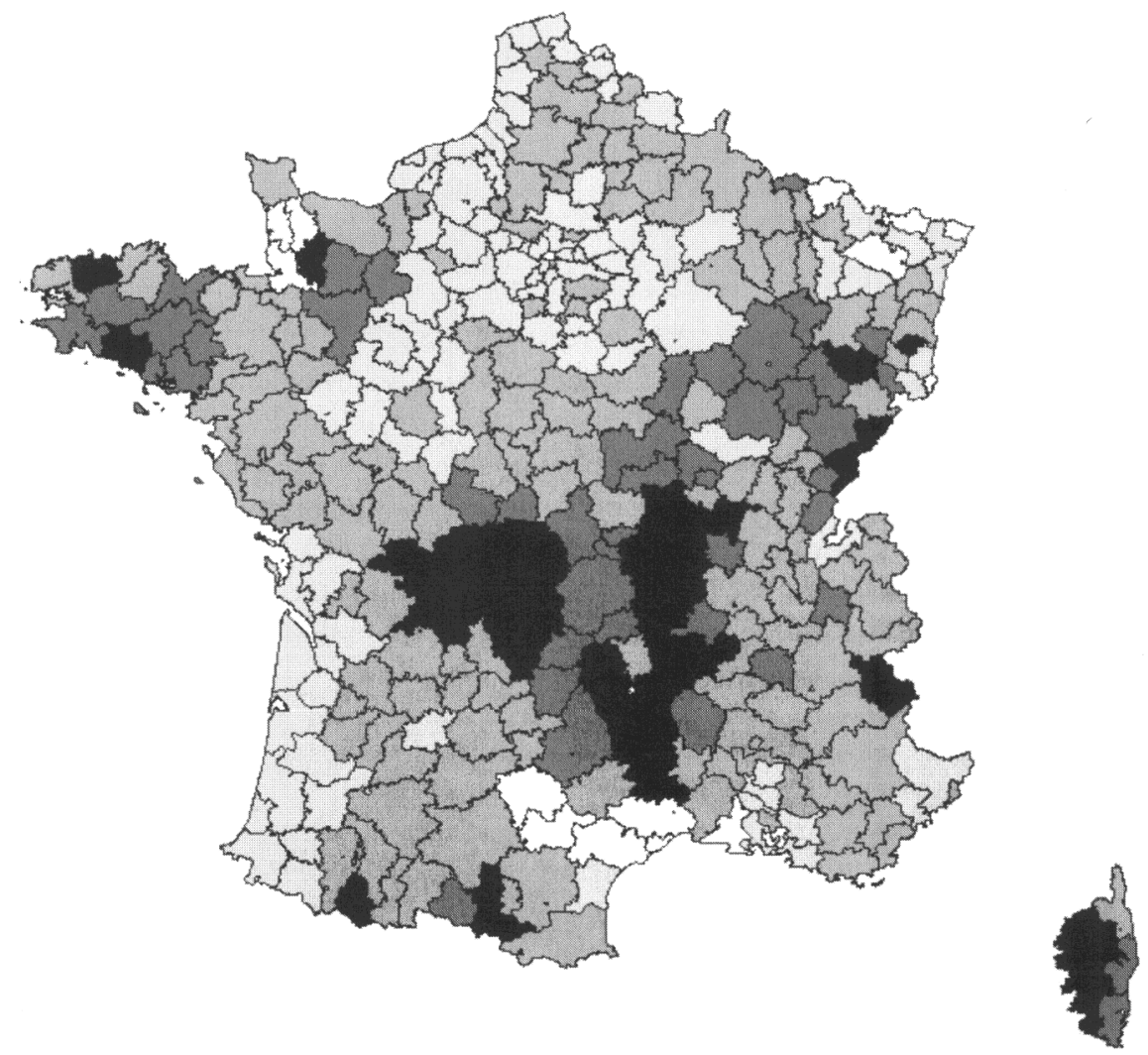

Concentrations en radon, en $\mathrm{Bq} / \mathrm{m}^{3}$ - Radon concentrations, in $\mathrm{Bq} / \mathrm{m}^{3}$ :

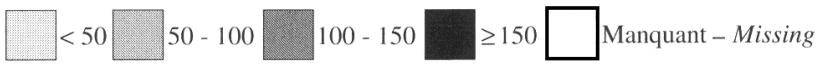

Figure 6-Moyennes arithmétiques des concentrations en radon par zone d'emploi, en Bq/m ${ }^{3}$, après correction pour la saison et le type d'habitat.

Arithmetic means of radon concentrations by "zone d'emploi", in Bq/ $\mathrm{m}^{3}$, after season and dwelling type corrections. 
de la dose efficace due au radon (suivant la formule de la publication 65 de la CIPR ; ICRP, 1993), aux rayonnements gamma d'origine tellurique et aux rayonnements cosmiques.

À partir des estimations réalisées, la population française reçoit une dose efficace annuelle moyenne due à la radioactivité naturelle de l'ordre de $2,2 \mathrm{mSv}$. Dans les départements français où une estimation conjointe de l'exposition au radon et aux rayonnements gamma et cosmiques a été possible (Figs. 2-5), les Landes est le département où la population est la moins exposée, tandis que la Lozère est le département où la population a l'exposition la plus élevée.

Les estimations de l'exposition de la population fournies dans cette étude confirment et renforcent celles des études antérieures sans correction pour le facteur saisonnier ou les caractéristiques d'habitat: la dose efficace annuelle résultant de l'exposition à la radioactivité naturelle, à l'exception de l'ingestion de l'eau et des aliments, passe d'environ $3 \mathrm{mSv}$ dans l'étude de Chartier et al. (1997) à $2,2 \mathrm{mSv}$ dans la présente étude.

\subsection{Applications épidémiologiques}

L'exposition de la population française aux rayonnements ionisants provient en majeure partie de la radioactivité naturelle. La France présente des variations géographiques importantes des niveaux d'exposition (Figs. 1-5). La moyenne nationale n'est donc pas l'indicateur de l'exposition de la population à la radioactivité naturelle le plus adéquat, notamment pour la mise en parallèle avec des données de surveillance sanitaire recueillies à des échelles géographiques plus fines, comme par exemple, les données de registres de cancer à l'Institut national de la santé et de la recherche médicale (INSERM) ou à l'Institut de veille sanitaire (InVS). Il est donc important et nécessaire de poursuivre les études sur les facteurs d'influence et de corriger les données pour disposer de l'indicateur le plus pertinent en fonction de l'objectif recherché.

De plus, il est possible d'estimer l'exposition de la population française à un niveau géographique plus fin que le département comme les zones d'emploi, ce qui est préférable dans un contexte épidémiologique, si les données de surveillance sanitaire sont disponibles à la même échelle. En effet, le découpage en zones d'emploi présente l'avantage d'être de plus en plus utilisé dans les études épidémiologiques et d'être plus pertinent, comme par exemple dans le cas de la mortalité par cancer broncho-pulmonaire (Rican et al., 1999 ; Salem et al., 1999).

Au regard des données sanitaires à mettre en parallèle, la dose efficace est souvent un indicateur peu pertinent. Dans la majorité des cas, les expositions à la radioactivité naturelle sont traitées séparément (radon, rayonnements gamma 
d'origine tellurique et rayonnements cosmiques), du fait de leur mode d'action différent (par exemple : inhalation des descendants du radon et risque de cancer du poumon). Les analyses portent donc généralement sur la relation entre un indicateur sanitaire et des concentrations ou des débits de dose. Néanmoins, dans le cas de l'analyse d'un cancer spécifique, la dose à l'organe pertinent (par exemple, dose à la moelle osseuse dans le cas de la leucémie) peut être calculée pour fournir un indicateur cumulant les trois types d'exposition naturelle (Rommens et al., 2001).

\section{Conclusion}

Les mesures effectuées lors des campagnes nationales constituent une importante source d'information : elles permettent d'étảblir une cartographie des niveaux de radioactivité naturelle due aux émanations de radon ou des rayonnements gamma sur le territoire français, et d'estimer l'exposition de la population. Les indicateurs de l'exposition de la population française reposent sur les concentrations en radon, les débits de dose des rayonnements gamma d'origine tellurique ou les doses dues aux rayonnements cosmiques après s'être affranchi des variations dues aux principaux facteurs d'influence. Ces indicateurs, pris individuellement ou dans leur ensemble, peuvent ainsi être analysés conjointement à des indicateurs de surveillance sanitaire, comme par exemple l'incidence des leucémies chez l'enfant, dans le cadre d'études écologiques.

Remerciements. Ce travail a été partiellement supporté par le Ministère de l'aménagement du territoire et de l'environnement, dans le cadre du programme de recherche « Environnement et Santé $1999 »\left(\right.$ convention $N^{\circ}$ AC014E).

\section{RÉFÉRENCES}

Arvela H. (1995) Seasonal variation in radon concentration of 3000 dwellings with model comparisons, Radiat. Prot. Dosim. 59, 33-42.

Axelson O., Fredrikson M., Akerblom G., Hardell L. (2002) Leukemia in childhood and adolescence and exposure to ionizing radiation in homes built from uranium-containing alum shale concrete, Epidemiology' 13, 146-150.

Baysson H., Tirmarche M. (2004) Exposition domestique au radon et risque de cancer du poumon : bilan des études cas-témoins, Rev. Epidemiol. Santé Pub. (sous presse).

Baysson H., Billon S., Laurier D., Rogel A., Tirmarche M. (2003) Scasonal correction factors for estimating radon exposure in dwellings in France, Radiat. Prot. Dosim. 104, 245-252.

Baysson H., Tirmarche M., Tymen G., Gouva S., Caillaud D., Artus J.C., Vergnenegre A., Ducloy F., Laurier D. (2004) Case-control study on lung cancer and indoor radon in France, Epidemiology (accepté).

BBC (1978) British Broadcasting Corporation, The people's activities and use of time. Audience Research Department. London. 
Beaugedin-Seiler K., Cessac B., Marot F., Chartier C., Deportes I., Métivier J.M., Morin A., Mourlon C., Vincent G. (2002) La banque de données CIBLEX, une compilation de paramètres d'exposition de la population française au voisinage d'un site pollué. Première rencontre nationale de la recherche sur les sites et sols pollués, bilan et perspectives, ADEME, 12 13 decembre, 2002, Paris.

Billon S., Caër S., Gambard J.P., Laurier D., Rannou A. (2002) Exposition de la population française aux rayonnements gamma tellariques. Réactualisation de la hase de données GAMMA-EXPO. Institut de Radioprotection et de Sûreté Nucléaire. Note technique SEGR/LEADS/02-36 indice 2 .

Billon S., Morin A., Caër S., Baysson H., Gambard J.P., Laurieı D., Tirmarche M. (2003) Evaluation de l'exposition à la radioactivité naturelle en France. Congrès National de Radioprotection, SFRP, Juin 1/-13, 2003, Montpellier (http://wwin.sfip.asso./\%/Montpellier2003/pdf/hillon.pdf).

Bochicchio F., Marocco D., Quarto M., Sera F., Farchi S., Forastiere F. (2002) Uncertainty of seasonal variations of radon concentration in dwellings and implications for radon policies and epidemiological studies. European IRPA Congress, October 8-11, 2002, Florence-Italy:

Buchli R., Burkart W. (1985) Main sources of indoor radon in the Swiss Central Alps. Sci. Tot. Environ. $45,425-432$.

Chartier M., Meslet C., Backe J.C. (1997) Une estimation de l'exposition de la population française aux rayonnements d'origine naturelle. Congrès SFRP, 18-20 juin, 1997, Avignon.

Darby S., Hill D., Doll R. (2001) Radon: a likely carcinogen at all exposures, Ann. Oncol. 12, 1341 1351.

Flodin U., Fredriksson M., Persson B., Axelson O. (1990) Acute myeloid leukemia and background radiation in an expanded case-referent study. Arch. Entirom. Health 45, 364-366.

Forastiere F., Sperati A., Cherubini G., Miceli M., Biggeri A., Axelson O. (1998) Adult myeloid leukaemia, geology, and domestic exposure to radon and gamma radiation: a case control study in central Italy, Occup. Environ. Med. 55, 106-1 10.

Gambard J.P., Mitton N., Pirard P. (2000) Campagne nationale de mesure de l'exposition domestique au radon IPSN-DGS. Bilan et représentation cartographique des mesures au ol janvier 2000. Institut de Protection et de Sûreté Nucléaire (http://wn'wirisn.fr).

Gunby J.A., Darby S.C., Miles J.C., Gieen B.M., Cox D.R. (1993) Factors aflecting indoor radon concentrations in the United Kingdom, Health Phws. 64, 2-12.

ICRP Publication 65 (1993) Protection against Radon-222 at home and at work, Ann. ICRP 23(2),

Idrish Miah M. (2001) Study on the variation of indoor gamma radiation in the Dhaka district, Radiat. Prot. Dosim. 95, 365-370.

Iyogi T., Ueda S., Hisamatsu S., Kondo K., Haruta H., Katagiri H., Kurabayashi M., Nakamura Y., Tsuji N. (2002) Environmental gamma-ray dose rate in Aomori Prefecture, Japan, Health Plus. 82, $521-526$.

Kendall G.M., Smith T.J. (2002) Doses to organs and tissues from radon and its decay products, $J$. Radiol. Prot. 22, 389-406.

Kim C.K., Lee S.C., Lee D.M., Chang B.U., Rho B.H., Kang H.D. (2003) Nationwide survey of radon levels in Korea, Health Phys. 84, 354-360.

Kitto M.E. (2003) Assessing radon concentrations in areas with few measurements, Environ. Monit. Ass. 83, 163-175.

Laurier D., Valenty M., Tirmarche M. (2001) Radon exposure and the risk of leukemia: a review of epidemiological studies, Health Phys. 81, 272-288.

Majborn B. (1992) Seasonal variations of radon concentrations in single-family houses with different sub-structures, Radiat. Prot. Dosim. 45, 443-447. 


\section{S. BILLON et al.}

Miles J.C. (2001) Temporal variation of radon levels in houses and implications for radon measurement strategies, Radiat. Prot. Dosim. 93, 369-376.

Mjönes L. (1986) Gamma radiation in Swedish dwellings, Radiat. Prot. Dosim. 15, 131-140.

Morin A., Backe J.C., Métivier J.M. (2002) L'exposition externe de la population française aux rayonnements cosmiques. IRSN, Note technique SEGR/SAER/99-52 - indice 4.

OPRI (2001) Office de protection contre les rayonnements ionisants, Rapport d'activité 2000 (http://www.oprifr/opri/html_opri/mesure/france.htm ou via http://www.irsn.fr).

Pinel J., Fearn T., Darby S.C., Miles J.C.H. (1995) Seasonal correction factors for indoor radon measurements in the United Kingdom, Radiat. Prot. Dosim. 58, 127-132.

Pirard P., Robé M.C., Roy M. (1998) Expositions par inhalation du radon atmosphérique. Le radon, de l'environnement à l'homme (H. Métivier, M.C. Robé, Eds) pp. 83-106, collection IPSN. EDP Sciences, Les Ulis.

Rannou A., Posny F., Guezengar J., Madelmont C. (1984) Study of natural irradiation in dwelling places in France, Radial. Prot. Dosim. 7, 317-320.

Rannou A., Gambard J.P., Brenot J. (1992) Campagnes de mesure de l'irradiation naturelle gamma et radon en France - Bilan de 1977 à 1990. IRSN, Note technique SEGR/LEADS/92-10.

Rican S., Salem G., Jougla E. (1999) Disparités géographiques de mortalité par cancers bronchopulmonaires en France et échelles spatiales d'analyse : intérêt du changement d'échelle en géographie de la santé, Rev. Epidemiol. Santé Pub. 47, 411-420.

Rommens C., Ringeard C., Hubert P. (2001) Exposure of red bone marrow to ionising radiation from natural and medical sources in France, J. Radiol. Prot. 21, 209-219.

Salem G., Rican S., Jougla E. (1999) Atlas de la santé en France. Volume 1: Les causes de décès. John Libbey Eurotext, Paris.

Sugier A., Hubert P. (2002) Dans le domaine des rayonnements ionisants, les données dosimétriques existantes sont-elles suffisantes ? Rev. Epidemiol. Santé Pub. 50, 13-26.

Tirmarche M., Rannou A., Mollie A., Sauve A. (1988) Epidemiological study of regional cancer mortality in France and natural radiation, Radiat. Prot. Dosim. 24, 479-482.

UNSCEAR (2000) United Nations Scientific Committee on the Effects of Atomic Radiation, Sources and effects of ionising radiation. UNSCEAR 2000 report to the general assembly, with scientific annexes. United Nations, New York.

Verger P., Hubert P., Cheron S., Bonnefous S., Bottard S., Brenot J. (1994) Use of field measurements in radon mapping in France, Radiat. Prot. Dosim. 56, 225-229. 